\title{
Percentagem de gordura de cavalos criados em região tropical
}

\author{
Percentage of fat in horses breed in tropical areas \\ Hélio Cordeiro Manso Filho ${ }^{1,2}$, Helena Emília Costa Cordeiro Manso', \\ Lúcia Maia Cavalcanti Ferreira' , Tito Alves Santiago², \\ Erika Korinfsky Wanderley ${ }^{2}$ \& José Mário Girão Abreu ${ }^{3}$
}

\begin{abstract}
RESUMO
Determinação da percentagem de gordura corporal (PG) pode contribuir para avaliar práticas de manejo nutricional e de treinamento. $\mathrm{O}$ objetivo do trabalho foi determinar a PG em equinos em condições tropicais. Froram analisados 121 cavalos, agrupados em: reprodução (subgrupos: jovens, éguas vazias, égua gestação I e II, égua lactação I e II e garanhão) e atletas (subgrupos: vaquejada e marcha). A PG foi avaliada a partir da camada de gordura pela ultrassonografia. Os resultados foram submetidos a ANOVA e o método de Tukey para animais de reprodução e o teste T para atletas. Demonstrou-se variação na PG no grupo reprodução, sendo que os jovens $(\sim 10,4 \%)$ apresentaram menor PG que o garanhão $(\sim 16,6 \%)$, égua vazia $(\sim 14,0 \%)$ e égua gestante I $(\sim 14,8 \%)$ e II $(\sim 15,5 \%)$ (p<0,05). Fêmeas lactantes obtiveram PG semelhante aos animais jovens. Também observou-se que animais que competem em vaquejada ( 10,8\%) possuem menor PG do que os de marcha $(\sim 14,7 \%)$ $(\mathrm{p}<0,05)$. A determinação da PG é importante para a compreensão das adaptações metabólicas durante as diferentes fases produtivas e/ou de treinamentos em condições tropicais de estabulação e criação, podendo ser importante para o estabelecimento de práticas de manejo adequadas nos grupos estudados.
\end{abstract}

Descritores: equinos, composição corporal, reprodução, exercício.

\begin{abstract}
Determination of fat percentage (PG) can be used as an indicator of management practices. The aim of this research was the determination of fat percentage (FP) in two different equine groups created in tropical conditions. 121 horses were analyzed and grouped in: reproduction group (subgroups: young, maiden mares, pregnant mares I and II, lactation mares I and II, stallion) and athlete group ("vaquejada" and "marcha"). The FP was measured by using fat thickness, which was measured by real-time ultrasound. The results were analyzed by ANOVA and Tukey test, also by T-test for athletic group. Results demonstrated that equines had FP associated with their activities. Young animals $(\sim 10,4 \%)$ had smaller FP when compared with stallion $(\sim 16,6 \%)$, maiden mares $(\sim 14,0 \%)$ and pregnant mares $(\sim 14,8 \%)$ and II $(\sim 15,5 \%)(\mathrm{p}<0,05)$. Young animals and lactating mares I and II had similar FP. Equines used for "vaquejada" $(\sim 10,8 \%)$ had smaller FP than animal used in "marcha" $(\sim 14,7 \%)(\mathrm{p}<0,05)$. FP characterization may be used to improve our knowledge about metabolic adaptation during different productive phases and/or exercise training in horses in tropical zones, and also could be important to establish correct management practices in those groups.
\end{abstract}

Keywords: equine, body composition, reproduction, exercise. 


\section{INTRODUÇÃO}

A criação intensiva de equinos de reprodução e de competição representa um significativo estresse sobre o sistema de controle energético dos cavalos, principalmente em condições tropicais. Estima-se que uma cavalo de $500 \mathrm{~kg}$ e com $5 \%$ de gordura possui 10 vezes mais energia acumulada na forma de gordura do que na forma de glicogênio, todavia ainda há poucos estudos que indiquem a percentagem de gordura em diferentes grupos de equideos [13]. Devido a esses fatos, tem sido estimulado a mensuração da percentagem de gordura corporal em equinos, para uma melhor compreensão das adaptações metabólicas, durante as diferentes fases produtivas e/ou durante treinamentos dos cavalos.

Diferentes métodos podem ser empregados para a determinação da massa de gordura, sendo que a determinação da percentagem de gordura corporal através da ultrassonografia é o mais simples e de fácil aplicação [5]. Há descrições da percentagem de gordura em animais de corrida, de éguas de reprodução, potros em crescimento e em animais idosos [1,6,8-10, 12], todavia não há informações sobre a percentagem de gordura corporal em animais que desempenham atividades físicas rotineiramente empregadas no nordeste brasileiro, como as provas de marcha e a vaquejada, e, também, para os animais de reprodução criados no sistema intensivo nessa região, que se caracteriza pelos longos períodos secos e baixa precipitação pluviométrica com temperaturas elevadas.

Com isso a determinação da percentagem de gordura corporal poderá contribuir para avaliar alguns aspectos do manejo dos cavalos, como o programa de arraçoamento e de treinamento, de forma objetiva e precisa. O objetivo do trabalho foi determinar a percentagem de gordura corporal (PG) em dois grupos distintos de equinos, sendo um de reprodução e outro de competição, em cavalos criados no nordeste brasileiro.

\section{MATERIAS E MÉTODOS}

A metodologia empregada neste trabalho foi aprovada Comitê de Ética do Departamento de Zootecnia, da Universidade Federal Rural de Pernambuco (CTA\#62/2007)

\section{Animais e sistema de produção}

Foram avaliados 121 animais, estabulados em 10 criatórios diferentes, que estavam num raio de $60 \mathrm{~km}$ do Recife (latitude 8`3'14"S; longitude 3452'51"W), com idades variadas e das raças: Campolina (3 fazendas), Mangalarga-Marchador (3 fazendas), Paint-Horse (1 fazenda) e Quarto-de-Milha (3 fazendas). Os animais estavam sob regime intensivo, no qual passam grande parte do dia em cavalariça, onde recebiam concentrados comerciais e forragens, sempre em conformidade com as suas necessidades [13]. As forragens eram basicamente capim elefante (Pennisetum purpureum), fornecido picado, em comedouros e 3 vezes ao dia; as pastagens dos potreiros eram compostas por forragens nativas, que durante o período seco do ano (setembro à abril), desaparecem quase que por completo. Sal mineralizado e água eram fornecidos ad libitum.

Com o intuito de facilitar as comparações entre os animais avaliados, eles foram agrupados em animais de reprodução e animais atletas. Em seguida, os animais de reprodução foram reagrupados em 7 grupos, sendo: animal jovem ( $<18$ meses), égua vazia, égua gestante I (2/3 iniciais da gestação), égua gestante II (1/3 final da gestação), égua lactação I (1/2 inicial da lactação), égua lactação II (1/2 final da lactação), e garanhão. Já os atletas, foram reagrupados em: cavalos de prova de marcha (resistência; $n=22$; raças Campolinas e MangalargaMarchadores) e cavalos de vaquejada (força e velocidade; $n=28$; raças Quarto-de-Milha e Paint-Horse). Esses atletas estavam treinando há mais de 4 meses e havia competido, nos últimos 2 meses, em eventos para cada raça.

\section{Determinação da percentagem de gordura}

Foi utilizado o método [16], no qual se utiliza a determinação da espessura da camada de gordura (CG) na garupa para determinação da percentagem de gordura corporal $(\mathrm{PG})[\mathrm{PG}=8,64+(4,7 \times \mathrm{CG})]$. Esse método consiste na colocação de um transdutor ultrasonográfico de $5 \mathrm{MHz}$ (Pie Medical ${ }^{\circledR}$ Linear Array Scanner 450), numa linha média entre a ponta do íleo e a do ísquio, a cerca de $10 \mathrm{~cm}$ da linha média do corpo e, por conseguinte, a vizualização da CG nessa região.

\section{Análise estatística}

Os resultados dos animais de reprodução foram submetidos a análise da variância e o método de Tukey foi utilizado para a comparação múltipla entre as médias, sendo o nível de significância estabelecido quando $\mathrm{P}<0,05$. Os resultados dos animais atletas foram analisados pelo teste-T, também com o nível de significância estabelecido, quando $\mathrm{P}<0,05$. Os resultados estão expressos em média +/- erro padrão. Em ambos os casos, utilizou-se o SigmaStat ${ }^{\circledR} 3.0$ para o sistema operacional Windows ${ }^{\circledR}$. 
Tabela 1. Camada de gordura e percentagem de gordura corporal nos equinos de reprodução criados em regiões tropicais, Recife, Pernambuco, 2007.

\begin{tabular}{|c|c|c|}
\hline \multirow[b]{2}{*}{ Categoria produtiva } & \multicolumn{2}{|c|}{ Avaliação } \\
\hline & $\begin{array}{l}\text { Espessura da camada } \\
\text { de gordura }(\mathrm{cm})\end{array}$ & $\begin{array}{l}\text { Percentagem da gordura corporal } \\
\qquad(\%)\end{array}$ \\
\hline Animal jovem $(n=14)$ & $0,37 \pm 0,05^{\mathrm{E}}$ & $10,38 \pm 0,26^{\mathrm{E}}$ \\
\hline Égua vazia $(n=15)$ & $1,13 \pm 0,19 \mathrm{ABCD}$ & $13,95 \pm 0,92 \mathrm{ABCD}$ \\
\hline Égua gestante I (2/3 iniciais) $(n=16)$ & $1,31 \pm 0,16^{\mathrm{ABC}}$ & $14,80 \pm 0,79 \mathrm{ABC}$ \\
\hline Égua gestante II (1/3 final) $(n=7)$ & $1,46 \pm 0,31{ }^{\mathrm{AB}}$ & $15,52 \pm 1,49$ АВ \\
\hline Égua lactante I $(1 / 2$ inicial $)(n=13)$ & $0,83 \pm 0,19$ ABCDE & $12,58 \pm 0,91 \mathrm{ABCDE}$ \\
\hline Égua lactante I (1/2 final) $(n=3)$ & $0,60 \pm 0,10 \mathrm{ABCDE}$ & $11,46 \pm 0,47 \mathrm{ABCDE}$ \\
\hline Garanhão (n=3) & $1,70 \pm 0,26^{\mathrm{A}}$ & $16,63 \pm 1,24^{\mathrm{A}}$ \\
\hline
\end{tabular}

Tabela 2. Camada de gordura e percentagem de gordura corporal nos equinos atletas criados em regiões tropicais, Recife, Pernambuco, 2007.

\begin{tabular}{lcc}
\hline \multirow{2}{*}{ Tipo do equino atleta } & \multicolumn{2}{c}{ Avaliação } \\
\cline { 2 - 3 } & $\begin{array}{c}\text { Espessura da } \\
\text { camada de gordura }(\mathbf{c m})\end{array}$ & $\begin{array}{c}\text { Percentagem da } \\
\text { gordura corporal (\%) }\end{array}$ \\
\hline Cavalo de Vaquejada ( $\mathrm{n}=28)$ & $0,464 \pm 0,003^{\mathrm{A}}$ & $10,822 \pm 0,121^{\mathrm{A}}$ \\
Cavalo de Marcha $(\mathrm{n}=22)$ & $1,305 \pm 0,115^{\mathrm{B}}$ & $14,771 \pm 0,540^{\mathrm{B}}$ \\
\hline Observações: médias, na coluna, seguidas pela mesma letra não diferem ao teste- $\mathrm{T}(\mathrm{P}>0,05)$.
\end{tabular}

\section{RESULTADOS}

Pela primeira vez, foi demonstrado que os animais, em condições tropicais e sob regime intensivo, acumulam gordura corporal em conformidade com o tipo de produção que desempenham, sendo que os animais jovens apresentaram menor $\mathrm{PG}$ que os grupos garanhão, égua vazia e égua gestante (I e II) $(\mathrm{P}<0,05)$ (Tabela 1). Não houve diferença entres os grupos garanhão, gestação I e II e lactação I e II $(P>0,05)$ sendo que a maior PG foi observada nos garanhões $(\sim 16,63 \%)$ enquanto que a menor nos animais jovens $(\sim 10,38 \%)$. Ainda foi observado (Tabela 2$)$ que os animais que competem em provas de vaquejada $(\sim 10,8 \%)$ possuem menor PG quando comparados com os de marcha $(\sim 14,7 \%)(\mathrm{P}<0,05)$.

\section{DISCUSSÃO}

Durante muito tempo, a importância do tecido adiposo foi relacionada com a proteção contra o frio e com a formação das membranas celulares, mas, com a determinação da importância da composição corporal para o manejo nutricional e atlético [13], passou-se a dar maior importância à mensuração da massa de gordura. Existem vários métodos para determinação da composição corporal e da PG, mas a utilização da ultrassonografia de tempo-real é um método simples e bastante preciso, substituindo a determinação da massa gordura pelo escore corporal [5]. O método pode ser empregado facilmente pelo especialista, fornecendo rápidos resultados e outro ponto que deve ser enfatizado é que, por ser um método de fácil compreensão e visualização, os resultados podem ser utilizados como um procedimento educativo, demonstrando aos cavalariços e aos proprietários/criadores as reais condições corporais dos animais avaliados naquele momento.

A determinação da PG nos animais de reprodução permite uma melhor avaliação das práticas de manejo. Em animais mantidos em regiões temperadas observou-se que há aumento da PG ao longo do anos, quando as fêmeas são mantidas vazias, sendo que essas reservas de energia podem ser um dos responsáveis pelo aparecimento do estro fora da estação de montas [2]. 
Entretanto, esse acúmulo de tecido adiposo tem sido associado ao aparecimento de enfermidades como resistência à insulina e à laminite em diferentes grupos de animais $[4,7,11]$. Nos animais avaliados no Nordeste, observou-se que as fêmeas em gestação e garanhões apresentaram percentagens de gordura maiores que as fêmeas em lactação, mas que os valores médios assemelham-se aos descritos na literatura [4,7]. Finalmente deve-se recordar que os equinos nascem com baixa PG, cerca de 2,6\% [14], e recentemente foi determinado em condições temperadas que as crias e os potros da raça Standardbred acumulam pouca gordura durante a fase de amamentação, com valores variando de $\sim 8,5 \%$, ao nascer, e 12,0\% antes da apartação aos 6 meses [8]. Esses resultados descritos em animais Standardbred são similares aos dos animais jovens em condições tropicais, que apresentaram intenso anabolismo, mesmo sob regime de estabulação intensiva.

Os animais atletas, que competem em diferentes modalidades, podem acumular gordura em quantidades diferentes, sendo que o consumo de oxigênio e as necessidades energéticas estão relacionados com a composição corporal [13]. No nordeste brasileiro, dois tipos de competições, a marcha e a vaquejada, são os responsáveis por mais de $80 \%$ dos eventos equestres, todavia há ainda poucos estudos sobre o metabolismo energético e as suas consequências para as práticas de manejo desses grupos de atletas. Na vaquejada, os cavalos deslocam-se ao galope $(\sim 25,0 \mathrm{~km} / \mathrm{h})$ em $3-5$ carreiras de 20-25 segundos, em dupla e com um bovino sendo puxado pela cauda, enquanto que na prova de marcha eles deslocam-se a marcha $(\sim 13,0 \mathrm{~km} / \mathrm{h}) \mathrm{du}-$ rante 30-40 minutos.

$\mathrm{O}$ aumento da $\mathrm{PG}$ pode significar aumento das reservas energéticas para os exercícios de resistência, mas não para os de corrida, pois a formação de glucose a partir de compostos originários do tecido adiposo leva algum tempo para ser efetivada $[3,11]$. Com isso, o maior acúmulo de gordura nos animais de marcha, quando comparado com os animais de vaquejada já era esperado, pois o primeiro grupo de atleta desenvolve atividades tipicamente aeróbicas e com isso necessita manter uma boa reserva de gordura para a produção de energia durante o exercício. Diferentemente, os de corrida apresentam baixa percentagem de gordura $(\sim 10,0 \%)$ e o tempo de corrida está correlacionado negativamente com a quantidade de gordura corporal [6]. Nesse caso, os resultados dos cavalos de vaquejada, que competem correndo inúmeras vezes, assemelhou-se aos descritos para esses últimos animais de corrida. Finalmente, deve-se observar que animais com o maior acúmulo de gordura (gordos ou obesos), quando estabulados em regiões quentes e úmidas necessitam de maior quantidade de energia para a manutenção, quando comparados com animais em bom estado corporal [15], porém ainda não há uma percentagem de gordura ideal para cavalos de diferentes atividades atléticas [13], mas, neste trabalho, ficou claro que deverá haver um padrão para os animais de marcha e de vaquejada que estão em plena competição.

Tomando-se de forma global os resultados desse experimento, observa-se que os animais jovens e os cavalos de vaquejada apresentam menores percentagens de gordura corporal quando comparado com os seus respectivos agrupamentos. A reduzida massa de gordura desses animais indica que os grupos de animais sujeitos a maiores desafios metabólicos (crescimento, lactação e competições de vaquejada) e, por conseguinte, maior nível de estresse energético, apresentaram menores acúmulos de gordura corporal.

\section{REFERÊNCIAS}

1 Fitzgerald B.P. \& McManus C.J. 2000. Photoperiodic versus metabolic signal as determinants of seasonal anaestrous in the mare. Biology of Reproduction. 63: 335-340.

2 Fitzgerald B.P., Reedy S.E., Sessions D.R., Powell D.M. \& McManus C.J. 2002. Potential signal medianting the maintance of reproductive activity during the non-breeding season in mares. Reproduction Supplement. 59: 115-129.

3 Hill J. 2007. Impacts of nutritional technology on feed offered to horses: A review of effects of processing on voluntary intake, digesta characteristics and feed utilization. Animal Feed Science and Technology. 138: 92-117.

4 Jeffcott L.B. \& Field J.R. 1986. Glucose tolerance and insulin sensitivity in ponies and standardbred horses. Equine Veterinary Journal. 18: 97-101.

5 Kearns C.F., McKeever K.H. \& Abe T. 2002. Overview of horse body composition and muscle architecture: Implications for performance. The Veterinary Journal. 164: 224-234.

6 Kearns C.F., McKeever K.H., Kumagai K. \& Abe.T. 2002. Fat-free mass is related to one-mile race performance in elite standardbred horses. The Veterinary Journal. 163: 260-266. 
7 Kronfeld D.S., Treiber K.H., Hess T.M. \& Boston R.C. 2005. Insulin resistance in the horse: Definition, detection, and dietetics. Journal of Animal Science. 83: E22-31E.

8 Manso Filho H.C., Watford M. \& McKeever K.H. 2004. Body composition in transition mares and suckling foals. In: $2 n d$ European Workshop on Equine Nutrition (Dijon, France). p. 88.

9 Manso Filho H.C., Costa H.E.C. \& Abreu J.M.G. 2008. Suplementação com aminoácidos modifica a composição corporal e parâmetros sangüíneos de eqüinos idosos. Revista do Conselho Federal de Medicina Veterinária. 43: 9-15.

10 Manso Filho H.C., McKeever K.H., Gordon M.E., Costa H.E.C., Lagakos W.S. \& Watford M. 2008. Changes in glutamine metabolism indicate a mild catabolic state in the transition mare. Journal of Animal Science. 86: 3424-3431.

11 McKeever K.H. 2002. Exercise physiology of the older horse. The Veterinary Clinics of North America (Equine practice). 18: 469-490.

12 McManus C.J. \& B.P. Fitzgerald. 2000. Effects of a single day of feed restriction on changes in serum leptin, gonadotropin, prolactin, and metabolites in aged and young mares. Domestic Animal Endocrinology. 19: 1-13.

13 NRC. 2007. Nutrient requirements of the horse. 6th Revised edn. Washington D.C.: National Academy Press, p.8.

14 Platt H. 1984. Growth of the equine foetus. Equine Veterinary Journal. 16: 247-252.

15 Potter G.D. 2002. Digestible energy requirements of horses for maintenance and work. In: V. Julliand \& W. Rosset-Martin (Eds). Nutrition of the performance horse. Eaap publications. No. III. Wageningen Academic (Dijon, France). pp.17-21.

16 Westerestervelt R.G., Stouffer J.R., Hintz H.F. \& Schryver H.F. 1976. Estimating fatness in horses and ponies. Journal of Animal Science. 43: 781-785. 